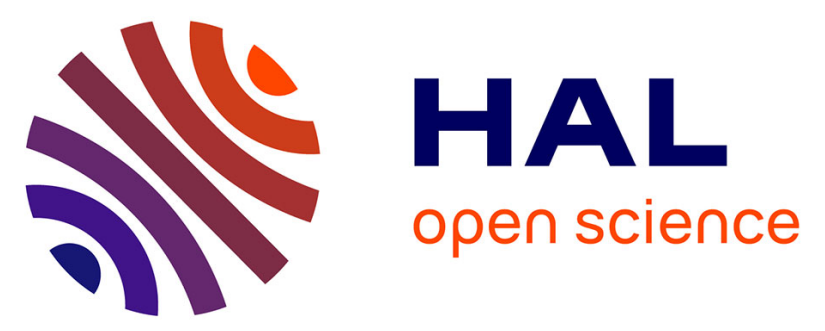

\title{
Broadband terahertz heterodyne spectrometer exploiting synchrotron radiation at megahertz resolution
}

Jean-Francois Lampin, O. Pirali, Z. Buchanan, Sophie Eliet, M.-A. Martin-Drumel, J. Turut, Pascal Roy, Francis Hindle, Gaël Mouret

\section{- To cite this version:}

Jean-Francois Lampin, O. Pirali, Z. Buchanan, Sophie Eliet, M.-A. Martin-Drumel, et al.. Broadband terahertz heterodyne spectrometer exploiting synchrotron radiation at megahertz resolution. Optics Letters, 2019, 44 (20), pp.4985-4988. 10.1364/OL.44.004985 . hal-02319308

\section{HAL Id: hal-02319308 \\ https://hal.science/hal-02319308}

Submitted on 8 Nov 2019

HAL is a multi-disciplinary open access archive for the deposit and dissemination of scientific research documents, whether they are published or not. The documents may come from teaching and research institutions in France or abroad, or from public or private research centers.
L'archive ouverte pluridisciplinaire HAL, est destinée au dépôt et à la diffusion de documents scientifiques de niveau recherche, publiés ou non, émanant des établissements d'enseignement et de recherche français ou étrangers, des laboratoires publics ou privés. 


\title{
Broadband terahertz heterodyne spectrometer exploiting synchrotron radiation at megahertz resolution
}

\author{
J.-F. Lampin ${ }^{1}$, O. Pirali 2,3 , Z. S. Buchanan ${ }^{4}$, S. Eliet ${ }^{1}$, M.-A. Martin-Drumel ${ }^{2}$, J. Turut ${ }^{1}$, P. $^{2}$ \\ ROY $^{3}$, F. HINDLE ${ }^{5}$, AND G. MOURET ${ }^{5}$ \\ ${ }^{1}$ Institut d'Electronique Microélectronique et Nanotechnologie (IEMN), Lille University, CNRS, Avenue Poincaré F-59652 Villeneuve d'Ascq Cedex, France \\ ${ }^{2}$ Institut des Sciences Moléculaires d'Orsay (ISMO), CNRS, Univ. Paris-Sud, Université Paris-Saclay, F-91405 Orsay, France \\ ${ }^{3}$ SOLEIL synchrotron, AILES beamline, l'Orme des Merisiers, Saint-Aubin, F-91190 Gif-sur-Yvette, France \\ ${ }^{4}$ Department of Chemistry, University of California, Davis, One Shields Ave, Davis, CA 95616, USA \\ ${ }^{5}$ Laboratoire de Physico-Chimie de l'Atmosphère (LPCA), Université du Littoral Côte d'Opale, Avenue Schumann F-59140 Dunkerque, France
}

Contact corresponding author(s): jean-francois.lampin@univ-lille.fr, olivier.pirali@synchrotron-soleil.fr, gael.mouret@univ-littoral.fr

Final accepted article compiled on November 8, 2019

Published in: Optics Letters 44, 4985 (2019) https://doi.org/10.1364/OL.44.004985

\begin{abstract}
A new spectrometer allowing both high resolution and broadband coverage in the terahertz (THz) domain is proposed. This instrument exploits the heterodyne technique between broadband synchrotron radiation and a quantum cascade laser (QCL) based molecular THz laser that acts as the local oscillator (LO). Proof of principle for exploitation for spectroscopy is provided by the recording of molecular absorptions of hydrogen sulfide $\left(\mathrm{H}_{2} \mathrm{~S}\right)$ and methanol $\left(\mathrm{CH}_{3} \mathrm{OH}\right)$ around 1.073 $\mathrm{THz}$. Ultimately, the spectrometer will enable to cover the 1-4 THz region in $5 \mathrm{GHz}$ windows at Doppler resolution.
\end{abstract}

Driven by the rapid technological innovation and availability of system components, modern laboratory molecular spectroscopy is undergoing rapid changes towards broadband and high resolution capabilities. Frequency-comb spectrometers now allow spectra to be recorded with unprecedented spectral resolution and sensitivity from the mid-infrared (mid-IR) to the extreme ultraviolet (see the review paper from Picqué and Hänsch [1]), while chirped-pulse instruments enable broadband investigations at centimeter and millimeter wavelengths (see Brown et al. [2] and a recent review from Park and Field [3]). Such breakthroughs are yet to impact the less mature terahertz (THz) domains (arbitrarily defined as $1-10 \mathrm{THz}$ ). Indeed, despite significant efforts to improve the coverage of this so-called "THz gap", molecular spectroscopy in this region usually entails a compromise between wide frequency coverage and high resolution. While high resolution capabilities are fast expanding toward the $\mathrm{THz}$ domain, both from lower and higher regions of the electromagnetic spectrum, the challenge of wide tunability remains. Quantum cascade lasers (QCLs), for instance, now enables high precision molecular spectroscopy in the $\mathrm{THz}$ range but tuning of the single-frequency THz QCLs is often limited to several $\mathrm{GHz}$ [4]. Improvement in high frequency electronic technology permits harmonic generation using Schottky diodes to reach frequencies as high as $4 \mathrm{THz}$ although at such frequency the components are not commercially available and only cover narrow parts of the spectrum with relatively low power [5]. Numerous opto-electronic solutions heterodyning two continuous wave optical sources have been exploited in the past decades (see e.g., Ref. [6]). This category of instruments has allowed many crucial measurements of $\mathrm{THz}$ spectra owing to excellent high-resolution but lacks of instantaneous broadband capabilities. Broadband $\mathrm{THz}$ measurements can be undertaken by time domain spectroscopy (TDS) [7], asynchronous optical sampling (ASOPS) [8], or Fourier-transform (FT) spectrometers, however at limited spectral resolution. The achievable resolution of TDS is, in principle, set by the mechanical delay line, typically to around $1 \mathrm{GHz}$. For ASOPS, the recording of spectra with a resolution better than the laser repetition rate is somewhat cumbersome, time consumming and only provides low sensitivity at the present time. FT spectrometers are the most widely used solution and the commercial instruments can provide an ultimate resolution of $30 \mathrm{MHz}$ [9]. As a consequence, the frequency metrology of molecular absorption is often limited by the apparatus function, defined by the optical path length 
difference of the instrument, rather than by the natural width of the transition (the Doppler width is around few $\mathrm{MHz}$ in this part of the electromagnetic spectrum).

Achieving combined broadband and high resolution spectroscopy in the THz domain would open new opportunities for molecular spectroscopy. It would enable the observation of, among others, fine physical effects (couplings) involving highlying energy levels of molecules, large amplitude motions, and the rotational structure of heavy molecules with dense spectra. For this latter point in particular, above $1 \mathrm{THz}$, high resolution (i.e., rotationally-resolved) spectroscopy is currently limited to molecules of 10-20 atoms in size [10]. The broadband $\mathrm{THz}$ gas phase observation at Doppler-limited resolution of large molecular species entirely relies on significant breakthroughs in the performance of $\mathrm{THz}$ instrumentation. Spectrometers based on the heterodyne technique appear as a promising approach to achieve this goal. In particular, important benefits from radioastronomy instrumentation allow the multiplexing of intermediate frequency (IF) signals using Fast Fourier Transform Spectrometers (FFTS) increasing by orders of magnitude the attainable instantaneous spectral bandwidth and the signal-tonoise ratio (SNR) of the IF spectra [11]. Alternatively, modern synchrotron facilities provide a stable, broadband, and relatively intense $\mathrm{THz}$ and far-infrared (far-IR) continuum exploited for molecular spectroscopy (see e.g., Ref. [9]) while the use of mid-IR QCL as the pump source of far-IR molecular lasers has tremendously increased the number of accessible far-IR lasing frequencies [12].

In this letter, we report on a significant advancement toward broadband, high resolution spectroscopy in the THz domain. We previously have exploited heterodyne techniques to characterize at high spectral resolution the synchrotron emissions in various operation modes of the machine [13]. Here, we demonstrate the successful development of a new generation of heterodyne instrument mixing the bright synchrotron continuum with a QCL-pumped molecular laser (local oscillator, LO) [12] and using a Hot Electron Bolometer (HEB, Scontel) mixer. As a proof of concept, we measured several pure rotation absorption lines of gas phase hydrogen sulfide $\left(\mathrm{H}_{2} \mathrm{~S}\right)$ and methanol $\left(\mathrm{CH}_{3} \mathrm{OH}\right)$ around $1.073 \mathrm{THz}$. By tuning to various LO frequencies, this spectrometer will ultimately allow to cover the $1-4 \mathrm{THz}$ region in few $\mathrm{GHz}$ windows.

This broadband $\mathrm{THz}$ heterodyne spectrometer exploits the synchrotron radiation extracted by the AILES beamline of the SOLEIL synchrotron facility, which serves as a far-IR continuum source, and a new generation optically pumped far-IR molecular laser acting as the LO (Fig. 1). In this study, the synchrotron radiation continuum was optically filtered by both a black polyethylene film and a 900-1100 GHz bandpass filter (Thorlabs) reducing the $3.4 \mathrm{~mW}$ full-range mean-power to about $1.6 \mu \mathrm{W}$ in the spectral range of interest. The beamline chambers, which were kept under secondary vacuum, and our set-up for measurements at atmospheric pressure were separated by a $50 \mu \mathrm{m}$ thick polypropylene window. After filtering, the beam was collimated using a $100 \mathrm{~mm}$ focal length plano-convex, aspheric polymethylpentene (TPX) lens and directed through a $55 \mathrm{~cm}$ long single-pass absorption cell equipped with $4 \mathrm{~mm}$ thick polytetrafluoroethylene (PTFE) windows. A $300 \mathrm{~mm}$ focal length PTFE lens, placed near the cell output prevented large divergence of the synchrotron beam. The far-IR molecular laser is an optimized version of the prototype developed at IEMN [12] with an output power on the order of $0.5 \mathrm{~mW}$ thanks to a new waveguide and an optimized output coupler [14]. Using ammonia $\left(\mathrm{NH}_{3}\right)$ as a gain medium, this laser generates intense LO frequencies resulting from pure inversion transition in the $v_{2}=1$ vibrationally excited state. The upper level $(J=3, K=3$, symmetry " $a$ ") of the lasing transition is optically pumped by a free-running QCL laser (Ad-Tech Optics) using intense absorption lines from the ground state. Stimulated emission in the $v_{2}=1$ level is obtained using a $50 \mathrm{~cm}$-long laser cavity built in a $6 \mathrm{~mm}$-internal diameter copper tubing, a flat brass mirror with a $1.2 \mathrm{~mm}$ diameter hole in the center, and an optimized output coupler. In this study, the $\mathrm{NH}_{3}$ pressure was set to about $15 \mu \mathrm{bar}$ and a 1,073,049 MHz LO frequency was produced. The instantaneous laser linewidth was measured to be narrower than 100 $\mathrm{kHz}$ [15] and the laser power to be of a few hundreds of $\mu \mathrm{W}$. The radiation was collected using a $200 \mathrm{~mm}$ focal length TPX lens and optically superposed to the synchrotron radiation exiting the absorption cell using a free-standing wire grid. Heterodyning of the two radiations was performed by the HEB optimized in the $0.3-4 \mathrm{THz}$ range and exhibiting a double side band noise temperature $\leq 2000 \mathrm{~K}$ at $2.5 \mathrm{THz}$. Its low-noise cryogenically cooled high electron mobility high-electron mobility transistor (HEMT) intermediate frequency (IF) amplifier (25 dB gain) allows for $2.5 \mathrm{GHz}$ instantaneous bandwidth. Owing to frequency refolding, the actual instantaneous frequency coverage is $5 \mathrm{GHz}$, and upper and lower sidebands can be discriminated by a slight tuning the laser frequency. The full IF bandwidth was amplified with an RF gain of $34 \mathrm{~dB}$ (Agile MwT AMT-A0032). The spectral analysis of the full IF bandwidth was realized using a FFTS (RPG-Radiometer Physics GmbH).



Fig. 1. Schematic representation of the broadband $\mathrm{THz}$ heterodyne spectrometer developed on the AILES beamline of the SOLEIL synchrotron.

In order to evaluate the performances of our spectrometer, the absorption spectra of hydrogen sulfide and methanol, two molecules exhibiting intense pure rotation transitions within a $5 \mathrm{GHz}$ bandwidth around the $1,073,049.6 \mathrm{MHz} \mathrm{LO}$ frequency were recorded using a respective pressure of 30 and $70 \mu$ bar in 
the absorption cell, and a $1 \mathrm{~s}$ acquisition time. Portions of the IF spectra showing the transitions recorded for each molecule are shown on Fig. 2. The spectra presented in Fig. 2 result from a post-processing adapted from Ref. [16] allowing to eliminate the contribution from the rectification (direct detection) of the modulation frequencies of the far-IR synchrotron continuum (Fig. 1). Due to this post-processing, intensity information can not currently be exploited. Several striking features of these plots have to be pointed out: i) the SNR ratio achieved in only $1 \mathrm{~s}$ of acquisition allows for the strongest transitions of both molecules to be observed; ii) the resolution of the instrument enables clearly the observation of two methanol transitions separated by $10 \mathrm{MHz}$ (a third of the maximum resolution achievable using commercial FTIR interferometers); and iii) the linewidths are close to the expected Doppler widths allowing for accurate frequency determination. Two main spurious effects (laser frequency fluctuations and saturation of the HEB mixer), however, are worthy of improvements and are as discussed in the following paragraphs.

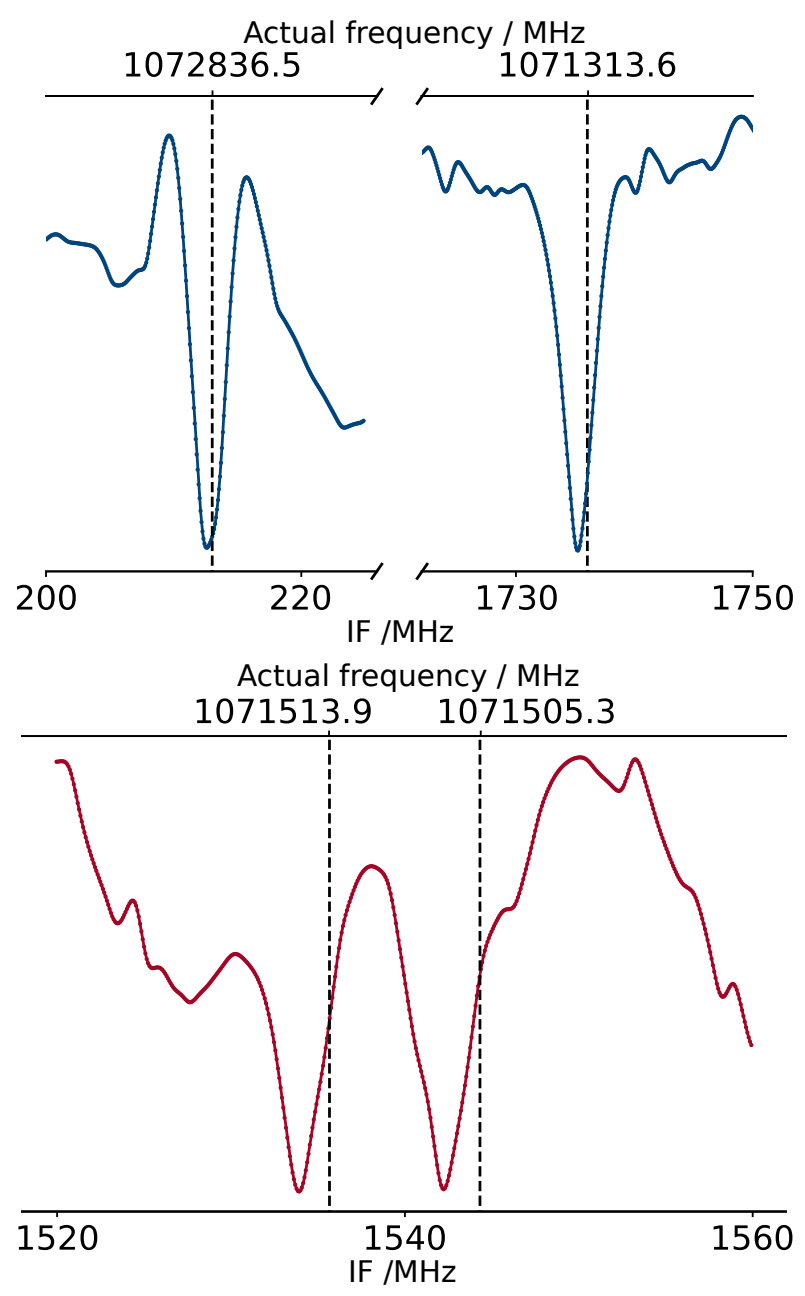

Fig. 2. Portions of the IF spectra extracted from the $2.5 \mathrm{GHz}$ full bandwidth for the two test-molecules $\mathrm{H}_{2} \mathrm{~S}$ (top trace, in blue) and $\mathrm{CH}_{3} \mathrm{OH}$ (bottom trace, in red). Both spectra were recorded in $1 \mathrm{~s}$ using the same 1,073,049 $\mathrm{MHz} \mathrm{LO}$ frequency. The vertical dotted lines indicate the literature frequencies.
Because of rapid temperature changes, the free-running QCL suffered of frequency fluctuations inducing relatively large variations of the $\mathrm{THz}$ laser intensity and frequency (within the Doppler width of the $\mathrm{THz}$ transition). In these conditions, the mixer response was significantly affected yielding strong evolution of the baseline between consecutive scans. As a consequence, baseline compensation between two scans (e.g., sample and reference spectra) is rather limited thus preventing from obtaining baseline flatness (Fig. 2). The actual noise level is very much smaller than it appears on the figure, but in the current configuration baseline fluctuations originating from the laser instability restrict the spectrometer performance. A second consequence of the $\mathrm{THz}$ laser frequency fluctuations is the observed shifts of the molecular transitions frequencies between successive scans. Since the center frequency of the successively recorded transitions can vary up to $1 \mathrm{MHz}$, averaging successive scans together yielded a broadening of the transitions. The FWHM of individual spectra is slightly larger the Doppler width of the transitions at room temperature, e.g., $2.9 \mathrm{MHz}$ measured for $2.2 \mathrm{MHz}$ expected in the case of the hydrogen sulfide transitions.

Further insights in our frequency metrology can be provided by comparing the line frequencies of the measured transitions with accurate values from the literature [17, 18]. Because of their relevance to many scientific fields, the high resolution spectra of both $\mathrm{H}_{2} \mathrm{~S}$ and $\mathrm{CH}_{3} \mathrm{OH}$ have been studied over a wide spectral region. Numerous articles have reported their pure rotation transitions that are summarized in databases such as the JPL [19] and CDMS [17] catalogs. In particular, $\mathrm{H}_{2} \mathrm{~S}$ pure rotation lines located above $1 \mathrm{THz}$ have been measured with $100 \mathrm{kHz}$ accuracy using a THz laser sideband spectrometer [20]. To the best of our knowledge the lines of methanol measured in the present work have not directly been measured. $\mathrm{Xu}$ et al. [21], however, reported a global treatment of a large data set including transitions observed at frequencies higher than $1 \mathrm{THz}$ with an accuracy of $50 \mathrm{kHz}$ thus frequency predictions reported in the CDMS database based on this work should be very accurate, to better than $100 \mathrm{kHz}$. The literature, when available, or predicted center line frequencies are reported in Fig. 2. Using our spectrometer, the line position accuracy is limited by the width of the absorption lines, their SNR ratio, and the LO frequency accuracy (both the numerical value used to convert IF into the actual frequencies and the laser frequency stability). Concerning the LO frequency value, Ref. [18] reported an experimental value of $1073049.5997 \mathrm{MHz}$ accurate to $50 \mathrm{kHz}$. The deviation of our measurements from the literature values is about 600 and $300 \mathrm{kHz}$ for the two lines of $\mathrm{H}_{2} \mathrm{~S}$ and 1.9 and 1.6 $\mathrm{MHz}$ for the $\mathrm{CH}_{3} \mathrm{OH}$ ones. While the small frequency difference of the hydrogen sulfide transitions can be imputed to the laser frequency fluctuations, it is possible that the larger value observed for methanol is rather a reflection of the uncertainty in the frequency prediction. Since in the present configuration the limited LO frequency accuracy also yielded limited SNR ratio and broader transitions, stabilization of the LO frequency has to be undertaken before further assessments on the actual frequency accuracy of the measured transitions are made.

The line profile of the hydrogen sulfide transitions is worth 
further discussion. While the line at $1,071,314 \mathrm{MHz}$ displays close to a Gaussian profile, the second component at $1,072,387 \mathrm{MHz}$ exhibits a second derivative-type profile. Since this line profile is only observed for one transition, we can exclude the Fourier Transform performed by the XFFTS or the baseline treatment to be responsible of it. Instead, we believe it may arise from saturation. Indeed, according to the JPL database [19], at room temperature this transition is supposed to be 6 times more intense than the lower frequency one. Since this intensity ratio is not reflected by our experimental measurements, such altered line profile of the stronger transition may be imputed to saturation effects.

Another source of noise in the spectra arises from the pulsed nature of the synchrotron emission which penalizes the operation of the spectrometer in two different aspects. First, direct detection of the pulse pattern provides intense frequency spikes on the IF spectrum . The rectification signal of the repetition rate $(352 \mathrm{MHz})$ as well as the revolution frequency $(846 \mathrm{kHz})$ give rise to two intense series of very sharp lines detected over the full IF bandwidth. Our post data treatment of the spectra was able to clean most of these spurious (see Fig. 1) but it prevents a rapid and more direct exploitation of the observations. Second, the relatively low average power (few hundreds of $\mathrm{nW}$ after filtering) translates into peak power of several watts for ps pulse duration. Such high power leads to rapid saturation of the HEB mixer and prevents the efficient mixing of the sources. Attenuation of such modulations of the $\mathrm{THz}$ continuum source is essential to improve further the sensitivity of the instruments.

Three major aspects of our spectrometer need to be upgraded: ultimate resolution, frequency coverage and SNR. From the LO side, it is clear that a better control of its frequency and intensity stability is highly demanded. Possibilities of locking the QCL frequency to standard frequencies is explored [15]. This new LO should provide the frequency metrology necessary to measure molecular adsorptions with sub-MHz accuracy on line position. It should also prevent the intense baseline variations detected on the IF spectrum which currently do not allow for longtime averaging and strongly decrease our ability to detect weak signals. A further benefit of the laser frequency stabilization will be the ability to finely tune its frequency over few $\mathrm{MHz}$, enough to discriminate upper and lower contribution around the LO frequency. Additionnally, a forthcoming laser cavity will soon permit to reach frequencies up to $6 \mathrm{THz}$, although the current HEB mixer will remain limited to $4 \mathrm{THz}$. A third important improvement should be performed to prevent HEB saturation. Time-stretching of the $\mathrm{THz}$ radiation by adapting technical concepts already developed for femto second lasers is currently under evaluation.

We demonstrated in this paper that the mixing of a pulsed source of $\mathrm{THz}$ continuum, here originating from synchrotron radiation, with a QCL-based molecular laser LO opens new possibilities for instantaneous broadband ultra-high resolution spectroscopy in this spectral region. The use of a HEB mixer together with a FFTS enables $5 \mathrm{GHz}$ of instantaneous bandwidth to be recorded. This spectrometer displays broadband capacities in two aspects: its frequency coverage (provided by the numerous available LO laser lines) and its instantaneous bandwidth. While all synchrotron-based far-IR spectroscopic stations over the world use FT spectrometers with an inherent limited spectral resolution, we demonstrated a new scheme of heterodyne detection for instantaneous broadband ultra-highresolution spectroscopy, by gathering a set of non-usual equipment developed for radioastronomical THz studies. Specifications of this first prototype have been obtained from well-known molecules, and spectral resolution of the order of $1 \mathrm{MHz}$ is clearly established, exceeding that of conventional FT instruments by at least one order of magnitude. Instrumental upgrades will enable an ultimate sub-MHz resolution (Doppler limited), a significant improvement in sensitivity (here, ability to record spectra over longer time period in order to improve the SNR and detect weaker signals), and a spectral coverage up to $4 \mathrm{THz}$. The $5 \mathrm{GHz}$ instantaneous bandwidth, combined with the numerous THz LO frequencies, allow heterodyne spectroscopy to be performed over a wide spectral region covering most of the $\mathrm{THz}$ range. This spectrometer be complementary to current instruments available at synchrotron facilities, in a spectral region still difficult to access.

Funding. This project received fundings from the Agence Nationale de la Recherche (ANR) (HEROES, ANR-16-CE300020-00); partial fundings from the LABoratoire d'EXcellence PALM (University Paris-Saclay) and from the SATT-Nord "TERAPOMPE" project.

Acknowledgment. The authors acknowledge SOLEIL for provision of synchrotron radiation under beamtime proposal 20180684 and we would like to thank J.-B. Brubach and K. Raddler for technical assistance. ZSB acknowledges support from the Chateaubriand Fellowship of the Office for Science \& Technology of the Embassy of France in the United States.

\section{REFERENCES}

1. N. Picqué and T. W. Hänsch. Frequency comb spectroscopy. Nat. Photonics, 13(3), 146-157 (2019). doi:10.1038/ s41566-018-0347-5.

2. G. G. Brown, B. C. Dian, K. O. Douglass, S. M. Geyer, S. T. Shipman, and B. H. Pate. A broadband fourier transform microwave spectrometer based on chirped pulse excitation. Rev. Sci. Instrum., 79(5) (2008). doi:10.1063/1.2919120.

3. G. B. Park and R. W. Field. Perspective: The first ten years of broadband chirped pulse fourier transform microwave spectroscopy. J. Chem. Phys., 144(20) (2016). doi:10.1063/1.4952762.

4. L. Consolino, F. Cappelli, M. Siciliani de Cumis, and P. De Natale. QCL-based frequency metrology from the mid-infrared to the $\mathrm{THz}$ range: a review. Nanophotonics, 8(2), 181 (2019). doi : $10.1515 /$ nanoph-2018-0076.

5. S. Yu, J. C. Pearson, B. J. Drouin, T. Crawford, A. M. Daly, B. Elliott, and T. Amano. Rotational spectroscopy of vibrationally excited $\mathrm{N}_{2} \mathrm{H}^{+}$ and $\mathrm{N}_{2} \mathrm{D}^{+}$up to $2.7 \mathrm{THz}$. J. Mol. Spectrosc., 314, 19-25 (2015). doi:10.1016/j.jms.2015.05.001.

6. S. Schlemmer, T. Giesen, F. Lewen, and W. G. High Resolution THz Spectroscopy and Applications to Astrophysics in "Frontiers of Molecular Spectroscopy". Elsevier Science (2009).

7. D. H. Auston and K. H. Cheung. Coherent time-domain far-infrared spectroscopy. J. Opt. Soc. Am. B, 2(4), 606-612 (1985). doi : 10 . $1364 /$ JOSAB. 2.000606.

8. Y.-D. Hsieh, Y. Iyonaga, Y. Sakaguchi, S. Yokoyama, H. Inaba, K. Minoshima, F. Hindle, Y. Takahashi, M. Yoshimura, Y. Mori, T. Araki, and T. Yasui. Terahertz comb spectroscopy traceable to microwave 
frequency standard. IEEE Trans. Terahertz Sci. Technol., 3(3, SI), 322-330 (2013). doi:10.1109/TTHZ.2013.2250333.

9. J.-B. Brubach, L. Manceron, M. Rouziéres, O. Pirali, D. Balcon, F. K. Tchana, V. Boudon, M. Tudorie, T. Huet, A. Cuisset, and P. Roy. In WIRMS 2009: 5th International Workshop on Infrared Microscopy and Spectroscopy with Accelerator Based Sources, volume 1214, page 81 (2010). doi:10.1063/1.3326356.

10. O. Pirali, M. Goubet, T. R. Huet, R. Georges, P. Soulard, P. Asselin, J. Courbe, P. Roy, and M. Vervloet. The far infrared spectrum of naphthalene characterized by high resolution synchrotron FTIR spectroscopy and anharmonic DFT calculations. Phys. Chem. Chem. Phys., 15(25), 10141-10150 (2013). doi:10.1039/c3cp44305a.

11. B. Klein, S. Hochgürtel, I. Krämer, A. Bell, K. Meyer, and R. Güsten. High-resolution wide-band fast fourier transform spectrometers. Astronom. Astrophys., 542, L3 (2012). doi:10.1051/0004-6361/ 201218864.

12. A. Pagies, G. Ducournau, and J. F. Lampin. Low-threshold terahertz molecular laser optically pumped by a quantum cascade laser. $A P L$ Photonics, 1(3) (2016). doi:10.1063/1.4945355.

13. S. Tammaro, O. Pirali, P. Roy, J. F. Lampin, G. Ducournau, A. Cuisset, F. Hindle, and G. Mouret. High density terahertz frequency comb produced by coherent synchrotron radiation. Nat. Commun., 6, 7733 (2015). doi:10.1038/ncomms8733.

14. A. Pagies, G. Ducournau, and J.-F. Lampin. Progress in continuous wave $\mathrm{THz}$ molecular laser optically pumped by a quantum cascade laser. 42nd International Conference on Infrared, Millimeter, and Terahertz Waves (IRMMW-THz), IEEE (2017). doi:10.1109/ IRMMW-THz.2017.8067232.

15. J. F. Lampin, A. Pagies, G. Santarelli, J. Hesler, W. Hansel, R. Holzwarth, and S. Stefano Barbieri. Quantum cascade laserpumped terahertz molecular lasers: frequency noise and stabilization using a 1560nm frequency comb. , submitted (2019).

16. X. Shen, L. Xu, S. Ye, R. Hu, L. Jin, H. Xu, and W. Liu. Automatic baseline correction method for the open-path fourier transform infrared spectra by using simple iterative averaging. Opt. Express, 26(10), A609-A614 (2018). doi:10.1364/OE.26.00A609.

17. C. P. Endres, S. Schlemmer, P. Schilke, J. Stutzki, and H. S. Müller. The Cologne Database for Molecular Spectroscopy, CDMS, in the Virtual Atomic and Molecular Data Centre, VAMDC. J. Mol. Spectrosc., 327, 95 - 104 (2016). New Visions of Spectroscopic Databases, Volume II. doi:https://doi.org/10.1016/j.jms.2016.03.005.

18. S. Yu, J. C. Pearson, B. J. Drouin, K. Sung, O. Pirali, M. Vervloet, M.-A. Martin-Drumel, C. P. Endres, T. Shiraishi, K. Kobayashi, and F. Matsushima. Submillimeter-wave and far-infrared spectroscopy of high- $J$ transitions of the ground and $v_{2}=1$ states of ammonia. $J$. Chem. Phys., 133(17) (2010). doi: \{10.1063/1.3499911\}.

19. H. Pickett, R. Poynter, E. Cohen, M. Delitsky, J. Pearson, and H. Müller. Submillimeter, millimeter, and microwave spectral line catalog. J. Quant. Spectrosc. Radiat. Transfer, 60(5), 883-890 (1998). doi:10.1016/ S0022-4073(98)00091-0.

20. S. P. Belov, K. M. T. Yamada, G. Winnewisser, L. Poteau, R. Bocquet, J. Demaison, O. L. Polyansky, and M. Y. Tretyakov. Terahertz rotational spectrum of $\mathrm{H}_{2} \mathrm{~S}$. J. Mol. Spectrosc., 173(2), 380-390 (1995). doi: 10.1006/jmsp.1995.1242.

21. L.-H. Xu, R. M. Lees, Y. Hao, H. S. P. Müller, C. P. Endres, F. Lewen, S. Schlemmer, and K. M. Menten. Millimeter wave and terahertz spectra and global fit of torsion-rotation transitions in the ground, first and second excited torsional states of $\mathrm{CH}_{3} \mathrm{OH}-\mathrm{C}-13$ methanol. $\mathrm{J}$. Mol. Spectrosc., 303, 1-7 (2014). doi:10.1016/j.jms. 2014. 06.005. 